\title{
CD300LG (Nepmucin) is differentially expressed in brain metastatic breast cancer.
}

Shahan Mamoor, MS ${ }^{1}$

1shahanmamoor@gmail.com

East Islip, NY USA

Brain metastases affect up to $34 \%$ of breast cancer patients treated with trastuzumab ${ }^{1}$. Limited treatment options are available for clinical control of brain metastatic breast cancer ${ }^{2-4}$. We mined published microarray data 5,6 to identify genes associated with metastasis to the brain in human breast cancer. This unbiased, global gene expression analysis identified differential expression of CD300LG as a transcriptional feature of brain metastasis in patients with breast cancer. Messenger RNA for CD300LG was present at significantly lower quantities in the brain metastatic tissues of patients with metastatic breast cancer. Additional microarray analysis revealed that CD300LG was also among the genes whose expression, transcriptome-wide, was most significantly different in primary tumors of the breast when compared to normal breast tissues. CD300LG is part of the transcriptional signature of human metastatic breast cancer.

Keywords: breast cancer, brain metastases, central nervous system metastases, CD300LG, nepmucin, systems biology of breast cancer, targeted therapeutics in breast cancer. 

central nervous system metastatic frequency of $34 \%$ for patients with breast cancer who had been treated with trastuzumab ${ }^{1}$. Unbiased approaches to describe the transcriptional landscape of brain metastases in patients with breast cancer are required. We mined published microarray data 5,6 to perform blind, global comparative transcriptome analysis of primary tumors and brain metastases in patients with human breast cancer. We identified CD300LG as among the genes whose expression was most significantly different in the brain metastases of patients with breast cancer as compared to primary tumors of the breast.

5

6

\section{Methods}

We used datasets GSE526045 and GSE1091696 for this global differential gene expression analysis of brain metastatic breast cancer in conjunction with GEO2R. GSE52604 was generated using Whole Human Genome Microarray 4x44K G4112F technology with $n=10$ normal breast tissues and $n=35$ brain metastases from patients with breast cancer, analysis performed using platform GPL6480. GSE109169 was generated using Affymetrix Human Exon 1.0 ST Array technology with $n=25$ normal breast tissue and $n=25$ tumors of the breast and; analysis was performed using platform GPL5175. The tissues are paired tissues ( 25 tumors matching 25 breast tissues from 25 patients). The Benjamini and Hochberg method of $p$-value adjustment was used for ranking of differential expression but raw $p$-values were used to assess statistical significance of global differential expression. Log-transformation of data was auto-detected, and the NCBI generated category of platform annotation was used. A statistical test was performed to evaluate whether CD300LG gene expression was significantly between primary breast tumors and brain metastases in humans with breast cancer using a two-tailed, unpaired t-test with Welch's correction. We used PRISM for all statistical analyses of differential gene expression in human breast cancer (Version 8.4.0)(455).

\section{Results}

We harnessed the power of blind, systems-level comparative transcriptome analysis to discover genes associated with metastasis to the brain in human breast cancer using published microarray data ${ }^{5,6}$.

\section{CD300LG is differentially expressed in the brain metastases of patients with brain metastatic breast} cancer.

We identified the gene encoding CD300LG, also known as nepmucin, as among the genes whose expression was most quantitatively different in brain metastases when compared to primary tumors of the breast in patients with breast cancer ${ }^{5}$. When sorting each of the genes expressed in brain metastases based on significance of difference in expression as compared to normal breast tissues, CD300LG ranked 18 out of 41093 total transcripts (Table 1), equating to $99.96 \%$ differential expression. Differential expression of CD300LG in brain metastases in patients with breast cancer was statistically significant (Table 1; $p=1.37 \mathrm{E}-18)$.

\section{CD300LG is differentially expressed in the primary tumors of patients with breast cancer.}

We queried a second microarray dataset 6 to assess whether differential expression of CD300LG was specific to brain metastasis or whether it could be observed in primary tumors of the breast. We found that CD300LG was also among the genes whose expression was most significantly different when comparing primary tumors of the breast to normal tissues from patients with breast cancer. When sorting each of the genes expressed in primary tumors of the breast based on significance of difference in expression as compared to normal breast tissue, CD300LG ranked 1142 out of 19076 total transcripts (Table 2), equating to $94.0 \%$ differential expression. 
Differential expression of CD300LG in primary tumors of the breast in patients with breast cancer was statistically significant (Table $2 ; p=1.37 \mathrm{E}-08$ ).

\section{CD300LG is expressed at significantly lower levels in the brain metastases of patients with brain metastatic breast cancer as compared to normal breast tissues.}

We obtained exact mRNA expression levels for CD300LG in primary tumors of the breast and in brain metastases to evaluate magnitude and direction of change in CD300LG gene expression in brain metastatic tissues. CD300LG was expressed at lower levels in brain metastases from patients with breast cancer as compared to primary tumors of the breast (Figure 1). Decreased expression of CD300LG in brain metastases as compared to normal breast tissues was statistically significant (Figure $1 ; p<0.0001$ ).

Thus, through comparative transcriptome analysis of benign, tumor and metastatic tumor tissues of patients with breast cancer using published microarray data 5,6 , we found here that CD300LG was among the genes whose expression was most significantly different in humans breast cancer: both when comparing brain metastases to normal breast tissues and when comparing primary tumors of the breast in patients with breast cancer, and that CD300LG was expressed at significantly lower levels in brain metastatic tissues as compared to normal breast tissue.

\section{Discussion}

We provided evidence here that CD300LG ${ }^{7,8}$ was among the genes whose expression was most significantly different in metastases to the brain as compared to normal breast tissue; we observed significantly decreased expression of CD300LG in brain metastatic tissues. One study has previously reported that nepmucin functioned in lymphocyte rolling and binding8; there are features that might enhance migration through the lymphatics during metastasis. However, we found that CD300LG was expressed at significantly lower levels in the brain metastatic tissues of patients with metastatic breast cancer. Another study has demonstrated specific expression of CD300LG on capillary endothelium9. CD300 family members are broadly described to function in lipid recognition ${ }^{10}$. CD300LG, also known as CD300G, unlike other CD300 family members, does not possess motifs indicative of stimulatory or inhibitory capacity like ITAMs or ITIMs ${ }^{10}$, but does contain a mucin-like domain and an IgV-like domain $^{7-10}$. Down-regulation of CD300LG may be important during tumor progression, from normal breast tissues to primary tumor of the breast to metastasis of any number of foreign sites, including the brain. 


\section{References}

1. Bendell, J.C., Domchek, S.M., Burstein, H.J., Harris, L., Younger, J., Kuter, I., Bunnell, C., Rue, M., Gelman, R. and Winer, E., 2003. Central nervous system metastases in women who receive trastuzumab-based therapy for metastatic breast carcinoma. Cancer, 97(12), pp.2972-2977.

2. Robson, M., Im, S.A., Senkus, E., Xu, B., Domchek, S.M., Masuda, N., Delaloge, S., Li, W., Tung, N., Armstrong, A. and Wu, W., 2017. Olaparib for metastatic breast cancer in patients with a germline BRCA mutation. New England Journal of Medicine, 377(6), pp.523-533.

3. Chang, J.W., Kuo, W.H., Lin, C.M., Chen, W.L., Chan, S.H., Chiu, M.F., Chang, I.S., Jiang, S.S., Tsai, F.Y., Chen, C.H. and Huang, P.H., 2018. Wild-type p53 upregulates an early onset breast cancerassociated gene GAS7 to suppress metastasis via GAS7-CYFIP1-mediated signaling pathway. Oncogene, 37(30), pp.4137-4150.

4. Tsukada, Y., Fouad, A., Pickren, J.W. and Lane, W.W., 1983. Central nervous system metastasis from breast carcinoma autopsy study. Cancer, 52(12), pp.2349-2354.

5. Salhia, B., Kiefer, J., Ross, J.T., Metapally, R., Martinez, R.A., Johnson, K.N., DiPerna, D.M., Paquette, K.M., Jung, S., Nasser, S. and Wallstrom, G., 2014. Integrated genomic and epigenomic analysis of breast cancer brain metastasis. PloS one, 9(1), p.e85448.

6. Harrell, J.C., Prat, A., Parker, J.S., Fan, C., He, X., Carey, L., Anders, C., Ewend, M. and Perou, C.M., 2012. Genomic analysis identifies unique signatures predictive of brain, lung, and liver relapse. Breast cancer research and treatment, 132(2), pp.523-535.

7. Umemoto, E., Tanaka, T., Kanda, H., Jin, S., Tohya, K., Otani, K., Matsutani, T., Matsumoto, M., Ebisuno, Y., Jang, M.H. and Fukuda, M., 2006. Nepmucin, a novel HEV sialomucin, mediates Lselectin-dependent lymphocyte rolling and promotes lymphocyte adhesion under flow. The Journal of experimental medicine, 203(6), pp.1603-1614.

8. Jiang, X., Wang, H., Li, Z., Wei, D., Yang, Y., Zheng, X., Bi, J. and Zhang, C., 2013. A monoclonal antibody against a novel sialomucin CD300LG. Monoclonal Antibodies in Immunodiagnosis and Immunotherapy, 32(2), pp.91-97.

9. Takatsu, H., Hase, K., Ohmae, M., Ohshima, S., Hashimoto, K., Taniura, N., Yamamoto, A. and Ohno, H., 2006. CD300 antigen like family member G: A novel Ig receptor like protein exclusively expressed on capillary endothelium. Biochemical and biophysical research communications, 348(1), pp.183-191.

10.Borrego, F., 2013. The CD300 molecules: an emerging family of regulators of the immune system. Blood, 121(11), pp.1951-1960. 


\begin{tabular}{r|l|l|l|l|l|l|}
\multicolumn{1}{l|}{ Rank } & ID & p-value & t & B & Gene & Gene name \\
\hline 18 & A_24_P305050 & $1.37 \mathrm{E}-18$ & -14.1820952 & 31.772893 & CD300LG & $\begin{array}{l}\text { CD300 molecule like } \\
\text { family member g }\end{array}$ \\
& & & & &
\end{tabular}

Table 1: Brain metastases from patients with metastatic breast cancer differentially express CD300LG when compared to normal breast tissue.

Rank of differential expression, probe ID, $p$-value with respect to differential expression, $\mathrm{t}$, a moderated $\mathrm{t}$ statistic, B, the log-odds of differential expression between the two groups compared, fold change (FC) of CD300LG expression between the brain metastases of patients with breast cancer and normal breast tissue, gene and gene name are listed in this chart.

PAGE 5 
Table 2: Brain metastases from patients with metastatic breast cancer differentially express CD300LG when compared to normal breast tissue.

Rank of differential expression, probe ID, $p$-value with respect to differential expression, $t$, a moderated tstatistic, B, the log-odds of differential expression between the two groups compared, gene and gene name are listed in this chart.

PAGE 6 


\section{CD300LG}

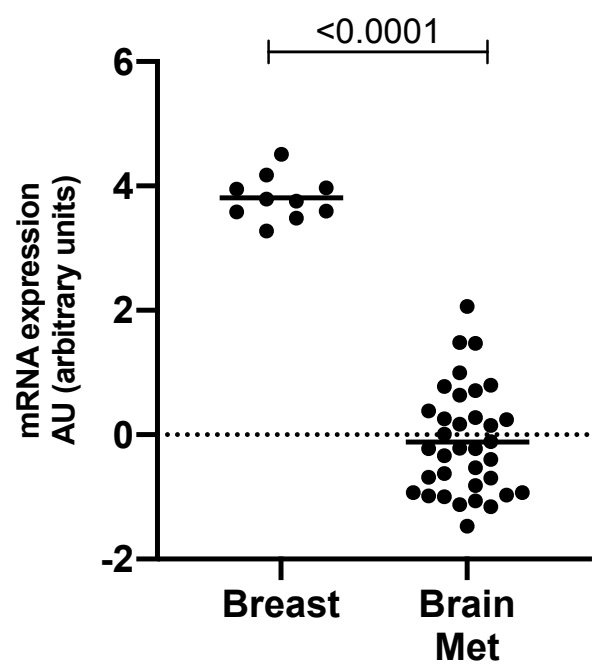

Figure 1: CD300LG is expressed at significantly lower levels in brain metastases as compared to normal breast tissues in patients with breast cancer.

The mRNA expression level of the CD300LG (CD300LG) in normal breast tissues (left) and in the brain metastases of brain metastatic breast cancer patients (right) is graphically represented here with mean CD300LG levels marked and the result of a statistical test evaluating difference in mRNA expression level between brain metastases and primary tumors of the breast, a $p$-value, listed above. 